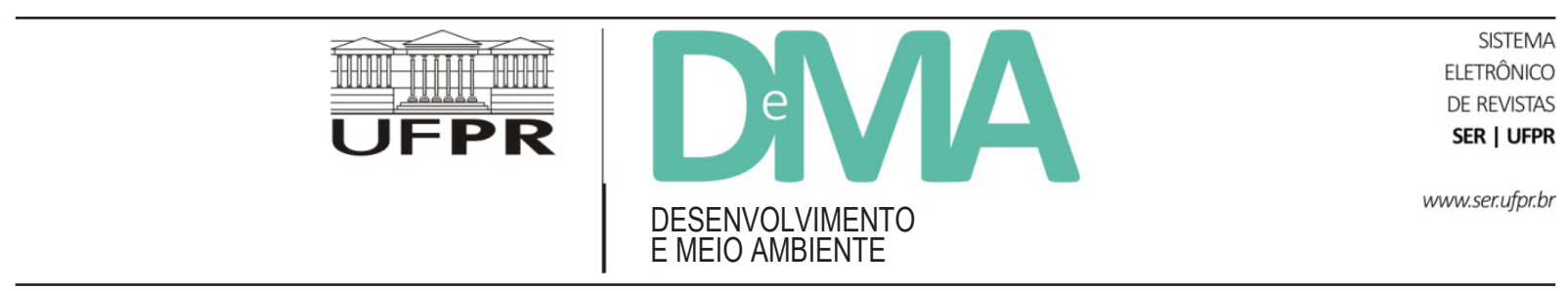

\title{
Ecología política latinoamericana y pensamiento crítico: vanguardias arraigadas
}

\author{
Ecologia política latino-americana e pensamento crítico: \\ as vanguardas enraizadas
}

\section{Political Ecology in Latin America and Critical Thinking: Rooted Vanguards}

\author{
Héctor ALIMONDA ${ }^{1^{*}}$ \\ (UFRRJ), Rio de Janeiro, RJ, Brasil. \\ *E-mail de contacto: hectoralimonda@gmail.com \\ Artículo recibido el 1 de octubre, 2015, versión final aceptada el 23 de desembro, 2015.
}

${ }^{1}$ Programa de Pós-Graduação de Ciências Sociais em Desenvolvimento, Agricultura e Sociedade, Universidade Federal Rural do Rio de Janeiro

RESUMEN: Este artículo intenta presentar la inserción de la Ecología Política que se está haciendo en América Latina (una elaboración intelectual reciente, que inclusive no ha consolidado su aceptación académica) con las tradiciones regionales de pensamiento crítico. Varios autores que analizan ese campo coinciden en señalar que la Ecología Política latinoamericana tiene características y dinámicas propias, diferenciadas de otras dos corrientes principales a nivel internacional, una de inspiración anglófona y otra vinculada con las tradiciones francesas. Como fundamento de esa particularidad, existe una historia diferenciada, donde se originan marcas estructurales en las sociedades latinoamericanas (la situación persistente de colonialidad de la región, la relevancia de la explotación de recursos naturales para exportación para la configuración de las estructuras sociales, la reproducción de la heterogeneidad estructural, la continuidad de actores subalternizados portadores de visiones alternativas de relaciones con la naturaleza). Pero también existe una tradición plural de reflexión teórico-política, la del Pensamiento Crítico, con la cual la Ecología Política presenta homologías. Recuperamos para referir esta relación la denominación de "vanguardias enraizadas" (o "arraigadas"), utilizada por el profesor Alfredo Bosi en relación a Mariátegui y su núcleo socialista del Perú de los años veinte.

Palabras clave: ecología política latinoamericana; pensamiento crítico latinoamericano; vanguardia arraigada.

RESUMO: O presente artigo visa apresentar a inclusão da Ecologia Política em curso na América Latina (que além de recente, sua elaboração tem resistência de aceitação acadêmica) com as tradições regionais do pensamento crítico. Diversos autores que analisam este campo coincidem em que a Ecologia Política latino-americana possui características e dinâmicas próprias, diferenciadas das outras correntes internacionais, uma de inspiração anglofônica e outra de tradição francesa. Para fundamentar esta particularidade em base a uma história 
diferenciada, na qual estão as marcas estruturais das sociedades latino-americanas: a situação persistente de colonialidade da região; a relevância da exploração dos recursos naturais para exportação e que configuram as estruturas sociais; a reprodução da heterogeneidade estrutural; a continuidade de atores subalternos, portadores de visões alternativas de relações com a natureza. Contudo, existe uma tradição plural de reflexão teórico-política, a do Pensamento Crítico, com a qual a Ecologia Política apresenta homologias. Para referir-nos a essa tradição, utilizamos a denominação de "Vanguardas Enraizadas" do Professor Alfredo Bosi quando se refere a Mariátegui e a seu núcleo socialista do Peru nos anos vinte do século passado.

Palavras-chave: ecologia política latino-americana; pensamento crítico latino-americano; vanguarda enraizada.

ABSTRACT: This article presents the inclusion of the ongoing Ecology policy in Latin America (besides being new, its development has academic acceptance resistance) with regional traditions of critical thinking. Several authors that analyze this field agree that Political Ecology of Latin America has its own characteristics and dynamics, distinguished from other international chains, an Anglophone inspiration and one of French tradition. To support of this particularity on the basis of a different story, in which are the structural brands of Latin American societies: the persistent situation of colonialism in the region; the relevance of the exploitation of natural resources for export and which shape the social structures; the reproduction of the structural heterogeneity; the continuity of subaltern actors, people with alternative views of relations with nature. However, there is a plural tradition of theoretical and political reflection, the Critical Thinking, with which the Political Ecology presents homologies. To refer us to this tradition, we use the name "Rooted Vanguard" from Professor Alfredo Bosi when referring to Mariátegui and his socialist core of Peru in the 20's of last century.

Key words: Latin American political ecology; critical Latin American thinking; rooted vanguard.

"La paradoja linda con una ironía típica del acto de escribir (en) América: sucesivas generaciones pueden negar las semejanzas literarias hasta el punto en el cual la negación misma pasa a constituir una semejanza". Doris Sommer, "Un romance irresistible: las ficciones fundacionales en América Latina", en Hommi Bhabha (compilador), Nación y Narración, 2010.

\section{Introducción}

A lo largo de las últimas dos décadas, se ha ido extendiendo en el campo intelectual internacional, y muy especialmente en América Latina, la denominada "ecología política". Originada básicamente a partir de elaboraciones académicas de las tradiciones anglo-sajonas (como los estudios de ecología humana, la geografía de Carl Sauer y los estudios culturales urbanos de Lewis Munford) y francesa (una sólida producción geográfica y antropológica, sumada a la tradición de economía regional), no registra, sin embargo, obras capitales de referencia que establezcan nuevos paradigmas o puntos de partida. El campo de la ecología política se fue estableciendo de forma molecular, a partir del surgimiento de nuevas preguntas y desafíos teóricos y prácticos, para los cuales no se contaba con respuestas al alcance de la mano. Por un lado, en diferentes áreas disciplinarias se fue produciendo un "giro eco-político", que abrió un doble programa de trabajo: la relectura de la propia tradición disciplinaria, a partir de nuevas claves, y el establecimiento de nuevos diálogos interdisciplinarios. Al mismo tiempo, una perspectiva de largo plazo puede verificar que ese "giro eco-político" fue también una respuesta (o, por lo menos, una recomposición intelectual para formular respuestas, sin darle a esta circunstancia una determinación necesaria) a la presencia de nuevos sujetos socio-políticos que, en las sociedades dichas avanzadas, asumían posiciones críticas y activas en relación a temas cruciales como el pacifismo en tiempos de guerra fría, la crítica al consumismo, las políticas de energía nuclear y la protección de ambientes naturales, muchas veces vinculadas con la formación de partidos "verdes". Hubo teóricos externos a la academia, como André Gorz, 
que intentaron una reformulación de la tradición crítica anti-capitalista, para dar cuenta de los nuevos desafíos ${ }^{1}$. A poco de andar por las últimas décadas del siglo XX, se fue haciendo evidente que también se estaba procesando una nueva dimensión de la relación capital/naturaleza, consolidando mecanismos de apropiación y explotación de los recursos planetarios de dimensiones y efectos desconocidos hasta entonces.

Fue a partir de allí que el término "ecología política” comenzó a circular por América Latina. En el momento actual, varios autores que han realizado balances de ese campo intelectual coinciden en caracterizar a la ecología política latinoamericana como una tradición político-intelectual específica, con una dinámica y estructura de enunciación que no tienen equivalente en otras áreas de elaboración político-intelectual (Kim et al., 2012; Delgado Ramos, 2013; Martínez Alier, 2014; Martin \& Larsimont, 2014).

Decía Joan Martínez Alier (2014), en ocasión de recibir el título de doctor honoris causa en la Universidad Nacional de Córdoba:

La ecología política estudia los conflictos socio-ambientales. Al mismo tiempo, el término designa un amplio movimiento social y político por la justicia ambiental que es más fuerte en América Latina que en otros continentes. Este movimiento lucha contra las injusticias ambientales en ámbitos locales, nacionales, regionales y globales (...) En América Latina la ecología política no es tanto una especialización universitaria dentro de los departamentos de geografía humana o de antropología social (al estilo de Michael Watts, Raymond Bryant, Paul Robbins) como un terreno de pensamiento propio de relevancia internacional, con autores muy apegados al activismo ambiental en sus propios países o en el continente como un todo.

Otro ejemplo, es la presentación realizada por Martin y Larsimont. Después de caracterizar la existencia de tres tendencias en el campo internacional de la ecología política, y de referirse a la political ecology anglosajona y a la ecologie politique francesa, se ocupan de la tercera, la ecología política latinoamericana.

Aunque en la EPLat se pueden identificar influencias y rasgos de origen más o menos disciplinares y académicos, sin duda sus características definitorias tienen que ver con un encuentro entre la tradición del pensamiento crítico latinoamericano y las vastas experiencias y estrategias de resistencia de los pueblos frente al saqueo y la "economía de rapiña". Mencionamos las reconocidas y tempranas contribuciones de José Carlos Mariátegui, Josué de Castro, Eduardo Galeano, entre muchas otras.

No obstante, desde fines de los años 90 se ha venido configurando una perspectiva diferenciada para el abordaje de las relaciones sociedad-naturaleza en la región. Probablemente lo diferencial en esta perspectiva sea su pretensión de hacerlo desde un "lugar de enunciación" latinoamericano. Esto implica reconocer los ámbitos teóricos y territoriales ajenos a las grandes tradiciones consolidadas de la geopolítica del pensamiento occidental. Asimismo, este lugar, de acuerdo a quienes convergen en el Programa de Investigación Modernidad/Colonialidad $(\mathrm{M} / \mathrm{C})^{2}$, se constituye en un posicionamiento ético, político y epistémico atravesado por la experiencia moderno/colonial pero que al mismo tiempo, se propone crear condiciones para la descolonización. El argumento central es que la marca de origen de lo latinoamericano se asienta en el trauma catastrófico de la conquista y la integración en posición subordinada y colonial en el sistema internacional. En este sentido entonces la EPlat da un lugar relevante a la experiencia histórica que implicó la colonización europea como ruptura de origen de la particular heterogeneidad y ambigüedad de las sociedades latinoamericanas (Alimonda, 2005). Esto, a su vez, supone la construcción de una historia ambiental de la región, en otras palabras, una "hermana siamesa" de la ecología política (Alimonda, 2005). ${ }^{3}$ Así la EPLat es una construcción colectiva en la que han confluido, no sin tensiones y debates, diversos autores de

\footnotetext{
${ }^{1}$ André Gorz utilizó explícitamente el término “ecología política”. Para Gorz, la defensa de la ecología del planeta podría ser recuperada por la lógica opresiva del capital, con una fisonomía eco-fascista. Por eso es que la ecología debería ser política, es decir, incluir una crítica de las formas de apropiación y explotación de la naturaleza y de las vidas humanas por parte del industrialismo, que no solamente controla la producción y el trabajo, sino que también impone los modelos de consumo y la formación de las subjetividades.

${ }^{2}$ El Programa M/C es un espacio contemporáneo de interlocución colectiva en y sobre América Latina donde participan referentes intelectuales como Arturo Escobar, Enrique Dussel, Aníbal Quijano, Walter Mignolo, Ramón Gosfoguel, entre otros.

${ }^{3}$ Guillermo Castro Herrera ha contribuido especialmente a consolidar la historia ambiental latinoamericana y a ponerla en diálogo con la ecología política. Claro está que es pertinente preguntarse en qué consiste lo latino de la ecología política latinoamericana, como lo hace Germán
} 
Latinoamérica poniendo énfasis en el estudio de las relaciones de poder, que son configuradas históricamente, como mediadoras de las relaciones sociedad/naturaleza (Martin \& Larsimont, 2014).

\section{Ecología política y pensamiento crítico}

Esta cita incluye los elementos que quisiéramos presentar aquí, examinando con un poco más de atención la propuesta de considerar a la ecología política latinoamericana como formando parte de la tradición del pensamiento crítico de la región. Lo haremos en la forma de una exposición muy general y necesariamente limitada, apenas como un intento de marcar los hitos epistémico-territoriales de estas tradiciones, en la forma del esbozo de una cartografía cognitiva (Jameson, 2002). Y teniendo como premisa metodológica el título de un artículo de Arturo Escobar (2005): "La cultura habita en lugares".

Es cierto que nuestra época (finales del siglo XX, primeros años del siglo XXI) configura características únicas, en especial en lo que se refiere a la relevancia que han adquirido las apropiaciones violentas de la naturaleza por parte del capital más concentrado, la aparición de la crisis climática global, el retroceso que este proceso implica en la perspectiva de las políticas de desarrollo y de derechos humanos, etc. Y también es obvio que las tradiciones más convencionales de las ciencias sociales internacionales no estaban preparadas para la respuesta analítica a estos nuevos desafíos, que suponen una radicalización de las tendencias destructivas de la modernidad, de las cuales ese pensamiento social es, por diferentes caminos, tributario.

Por este motivo, es frecuente encontrar la opinión de que la ecología política (y también la historia ambiental, consecuentemente) aparecerían como una novedosa construcción analítica, demandada por las características de la nueva crisis global y por los silencios de la teoría social y política convencional. Es perfectamente legítimo coincidir con esta posición.
Sin embargo, me gustaría invertir de alguna forma los términos del debate, no para oponerme a esta posición, saludablemente iconoclasta, pero por lo menos para asegurar más densidad a la posición crítica actual. Las diferentes generaciones intelectuales y políticas que se erigen en posiciones críticas sostienen razonablemente que su época es única y desafiadora, y que se enfrentan con problemas sustanciales que la diferencian de las generaciones anteriores. Pero creo que estas posiciones se refuerzan cuando, en la perspectiva de una geopolítica del conocimiento, reconocen raíces (reelaboradas a partir de nuevas lecturas, desde luego) en fuertes tradiciones del propio ámbito histórico-territorial.

A partir de un punto de vista personal, entonces, pero que se refiere en parte al camino recorrido y a mis referencias intelectuales presentes, creo que el "lugar de enunciación" plural y colectivo que ha ido constituyendo a (y constituyendo en) la ecología política latinoamericana tiene que ver, justamente, con la caracterización que el gran intelectual brasileño Alfredo Bosi (1992) hizo en relación a José Carlos Mariátegui y sus compañeros de generación intelectual y política: "la vanguardia enraizada".

En ese sentido, me gustaría poder pensar a esa ecología política como una elaboración político-intelectual de vanguardia, que intenta con angustia responder a los tremendos desafíos que la época presenta para los pueblos de nuestro continente, reconociendo la necesidad ineludible de criticar los presupuestos civilizatorios de la modernidad y del desarrollo convencionales. Para hacerlo, debe echar mano de todos los recursos posibles, que pasan en gran parte por la tarea paradigmática de actualizar sus repertorios de acción y de pensamiento, al mismo tiempo que debe intentar recuperar la pluralidad de herencias populares y críticas que la precedieron.

Vanguardia, pero arraigada en las determinaciones de su época y en las particularidades (llamemos) geopolíticas de sus puntos de vista. Vanguardia arraigada como lo fueron la generación de la Reforma Universitaria y de José Carlos Mariátegui, o lo mejor de los modernistas brasileños. Como lo ha sido en sus mejores momentos el pensamiento crítico latinoamericano.

Palacio (2012) en relación a la historia ambiental, sobre todo cuando se destaca la importancia de las herencias indígenas. Pero aceptemos por ahora las convenciones establecidas.

${ }^{4}$ Desde luego que coincidimos con Boaventura de Souza Santos cuando defiende al trabajo intelectual que se piensa como de "retaguardia". 
Pido que se me permita una referencia a mi experiencia personal. Cuando asumí la coordinación del Grupo de Trabajo en Ecología Política de CLACSO, en el año 2000, me pareció que una parte de la tarea por delante era intentar establecer un entretejido, en el peor de los casos para poner en contacto a intelectuales de diferentes países de la región que se desconocían mutuamente, en el mejor de los casos para intentar construir una comunidad de enunciación. Pero había también otra tarea, que a su vez tenía dos aspectos necesarios y complementarios, en la misma perspectiva estratégica: el fortalecimiento de nuestra propuesta justamente a partir de la creación de un espacio posible de diálogo con tradiciones anteriores de pensamiento social y político.

Por un lado, en tanto proyecto crítico de ciencias sociales, había que ajustar cuentas con el pasado de esas disciplinas. El blanco fundamental, en este caso, me pareció el marxismo, como la tradición crítica más establecida e institucionalizada en las ciencias sociales, pero siempre teniendo en cuenta que ese marxismo "internacional" (que venía de un intenso proceso de refundación/actualización a partir de los años setenta) ni agotaba la pluralidad de críticas anticapitalistas ni, en las condiciones latinoamericanas, siquiera era siempre anti-capitalista ${ }^{5}$. Por eso era necesario someterlo a una lectura crítica profunda, que permitiera recuperar dimensiones capaces de nutrir nuestra reflexión (no necesariamente desde lugares centrales o consolidados en esa tradición) y deshacernos de lo demás, los “ídolos", que en la mejor de las hipótesis sólo servían para reforzar la razón indolente, no apenas para darnos respuestas viejas, sino para desalentar la propia posibilidad de formular nuevas preguntas.

Al mismo tiempo, el ejemplo de la lectura eco-política de José Martí desarrollada por Guillermo Castro Herrera y el descubrimiento de una tradición de pensamiento eco-político brasileño desde el siglo XVIII, presentado por José Augusto Pádua, me mostraron que también teníamos una tarea decisiva en relación a los orígenes y las identidades del pensamiento latinoa- mericano. Nuestra crítica vendría a ser más potente justamente si podía arraigarse en una tradición, si podíamos reconocernos (como en el grupo sanguíneo o en la fisonomía de nuestros antepasados) en la genealogía del pensamiento crítico latinoamericano. De allí que propongo pensar a la constitución del lugar de enunciación de la ecología política latinoamericana en continuidad con ese pensamiento.

En primer lugar, el pensamiento crítico latinoamericano y la ecología política comparten como punto de partida la duda sobre nuestra identidad y la búsqueda ansiosa de las claves de la misma. En ese caso, sólo tenemos certidumbre de que no somos iguales a los países ejemplos tutelares de modernidad y desarrollo. Tampoco lo somos, dicho sea de paso, en relación a sociedades de otros continentes, donde la destrucción de culturas tradicionales por parte del eurocentrismo modernizante no llegó a los extremos de América. En Asia o en África hay sociedades que pueden regresar a los legados de su pasado, en América eso es más difícil. Esta desconfianza, claro, se extiende a los instrumentos teóricos, conceptuales y metodológicos a partir de los cuales poder pensarnos, en tanto herramientas tributarias de la modernidad eurocéntica.

Segundo, esta búsqueda de identidad sólo puede encontrar respuestas recurriendo al examen de nuestro pasado. De allí el recurso a la perspectiva histórica de interpretación, presente en todo el ensayismo latinoamericano, y también en la ecología política, en su entronque con la historia ambiental. Al hacerlo, surge como evidente el papel jugado en la definición de esa identidad por el proceso de la conquista ibérica, destruyendo a las civilizaciones nativas y nos incorporando a la modernidad/colonialidad en posiciones subordinadas. A partir de allí, y con la continuidad de la colonialidad y del proyecto que la modernidad tuvo para nosotros se originan nuestra particular heterogeneidad estructural, que se manifiesta en la economía, la sociedad, la cultura, la memoria, las identidades, la subordinación sistemática de poblaciones excluidas de la ciudadanía, las dificulta-

\footnotetext{
${ }^{5}$ Como decía Pancho Aricó, con la sacralización del aspecto "progresista" del desarrollo de las fuerzas productivas, El Capital fue en América Latina más el libro de la burguesía que del proletariado. "Por isso, uma obra que era concebida por Marx como o maior golpe teórico contra a burguesia, converteu-se, nos países atrasados, no livro dos burgueses, isto é, no mais solido fundamento para a aceitação da necessidade e progressividade do capitalismo tal como se configurou concretamente na Europa ocidental" (Aricó, 1982, p. 62).
} 
des para constituir un régimen político republicano que efectivamente funcione, y un Estado Nacional capaz de actuar legítimamente, la persistente actitud colonial también en lo que se refiere a la naturaleza, a la orientación de nuestras economías en la dirección del mercado mundial, en cadencias cíclicas de euforia y destrucción, y muchos otros males.

Tercero, al proceder a la enunciación de sus puntos de vista más generales, tanto el pensamiento crítico como la ecología política latinoamericana lo hacen en referencia a una realidad geo-histórica común, en la que se reconocen, aún cuando estén tratando de una sociedad nacional en particular. Para poner ejemplos, cuando José Carlos Mariátegui escribía los Siete Ensayos de Interpretación de la Realidad Peruana está claro que su discursividad iba acompañando la evolución peruana en los diferentes temas de su repertorio, pero también que por detrás hay un texto agazapado donde su análisis se interroga sobre la identidad y la historia de toda la región. De hecho, explícitamente, usa la comparación con la Argentina como recurso interpretativo (aunque en este punto sea demasiado optimista). De la misma forma, cuando cualquier latinoamericano lee los trabajos de Josué de Castro sobre el hambre en Brasil es interpelado, al mismo tiempo, por la realidad del hambre y las carencias sociales en su propio país. La generalización de la ofensiva por el despojo ambiental y la "acumulación por desposesión” en toda la región es tal que la pluralidad de libros que coleccionan estudios de caso de conflictos ambientales, en especial en temas como la gran minería, presentan situaciones que parecen reproducirse con las mismas características generales en todos los países (de hecho, muchas veces involucran a las mismas empresas).

Cuarto, entre el pensamiento crítico y la ecología política que se hace en América Latina existe una común desconfianza hacia el instrumental teórico y metodológico de las ciencias sociales convencionales. Se exhibieron y se exhiben dudas sobre su validez y aplicabilidad a las particularidades de nuestras realidades, sobre su vinculación con perspectivas finalistas de la historia, que desde el comienzo nos ponen en lugar de "casos desviados" en la marcha del progreso, sobre su dificultad, siendo que han sido elaboradas a partir de los repertorios de la modernidad, para interpelar a nuestras poblaciones desde posiciones de respeto y autonomía. Por esta causa, las herramientas metodológicas del pensamiento crítico y de la ecología política acostumbran presentar un destacado sesgo anti-positivista, y las fuentes son con frecuencia poco convencionales, pero de ellas se espera que puedan fundamentar con mayor pertinencia argumentaciones críticas al orden establecido. Con frecuencia, existen interlocuciones y articulaciones discursivas con tradiciones políticas populares subalternizadas. La ecología política tiene vinculaciones con el activismo socio-ambiental, en diferentes escalas.

Quinto, y por último, tanto el pensamiento crítico latinoamericano como la ecología política elaborada en la región se localizan en una posición fronteriza en relación a los sistemas de conocimiento establecidos. A pesar de co-partícipe del espíritu de la Reforma Universitaria, Mariátegui podía definirse como "no universitario, $e$ inclusive anti-universitario", aunque hubiera dirigido las Universidades Populares González Prada. En Brasil, fuera o dentro de la universidad, la convivencia de Caio Prado Jr. o de Darcy Ribeiro con la institucionalidad académica nunca fue fácil. De la misma forma, a pesar del notable impulso que ha tenido en la región, la ecología política fue creciendo en los márgenes de las estructuras universitarias, donde, a pesar de las apelaciones a la interdisciplinariedad, aún resisten los recortes disciplinarios como fundamento de estructuras establecidas de poder institucionalizado.

\section{El caso del Grupo de Trabajo de CLACSO}

Claro está que la "biodiversidad académica" que prolifera alrededor de la ecología política tiene su correlato en la dimensión ambiental de la crisis global, y especialmente en la expansión aceleradísima de los movimientos hegemónicos por la apropiación de la naturaleza latinoamericana, la reconfiguración de territorios y los conflictos desiguales en los que resisten las poblaciones. Muchas veces se tiene la sensación de que nuestra tarea intelectual está "llegando tarde" frente a las dimensiones de esta ofensiva. Sin embargo, a la distancia de una década y media, quizás pueda decirse que la creación del Grupo de Trabajo en Ecología Política, por 
parte del Comité Directivo de CLACSO, en octubre de 2000, tuvo un carácter anticipatorio destacable ${ }^{6}$.

Por un lado, a partir de sus reuniones anuales, realizadas en diferentes países, y de las ediciones de sus libros colectivos se fue construyendo un espacio común de intercomunicación entre intelectuales de la región, que encontraron la posibilidad de una elaboración conjunta y de una comunidad de enunciación sobre la situación socio-ambiental de sus propios países conjugada con la perspectiva regional, en un todo de acuerdo con las mejores tradiciones del pensamiento latinoamericano. Se fue originando así una "comunidad de pares", una red efectivamente vigente de relaciones interpersonales convocadas por preocupaciones y ansiedades comunes, que acabó demostrando que, además de su importancia estratégica intrínseca, tuvo un efecto multiplicador eminente en los medios académicos e intelectuales de la región.

Por otra parte, el grupo siempre interactuó con instituciones y movimientos colectivos que protagonizaban conflictos socio-ambientales manifiestos o latentes. Casi todas las reuniones, organizadas como prevé el reglamento por instituciones académicas de la red de CLACSO, contaron con la presencia de representantes de esos movimientos. El caso más destacado fue la reunión de Lima, en junio de 2009, pocos días después de los dramáticos hechos de Bagua. Invitados provenientes de movimientos sociales estaban heridos (Santiago Manuin, internado con cinco balazos) o prófugos (como Alberto Picanzo, asilado en la embajada de Nicaragua) pero la reunión tuvo una asistencia considerable, y sirvió para el establecimiento de un espacio público, donde representantes de esos movimientos, activistas e intelectuales peruanos y latinoamericanos discutieron sobre los acontecimientos. Esa reunión dio origen a dos libros publicados por CLACSO, uno conteniendo informaciones, cronología, testimonios y análisis sobre los hechos de Bagua (Alimonda et al., 2009, La Amazonía rebelde - Perú 2009, aparecido poco tiempo después de la reunión, y que llegó a estar, en febrero de 2010, en quinto lugar en las ventas de libros de no-ficción en la tradicional librería El Virrey, de Lima) y otro, en la colección Grupos de Trabajo de CLACSO, La naturaleza colonizada - Ecología Política y Minería en América Latina (Alimonda, 2011), dedicado al tema de la minería en Perú, incluyendo estudios de caso en otros países y artículos teóricos.

La perspectiva de contacto y retroalimentación con las resistencias populares vigentes tenía para el GT, claro está, un profundo significado epistemológico de largo alcance: en primer lugar, construir el conocimiento de la Ecología Política latinoamericana recuperando las voces y los saberes silenciados durante demasiado tiempo por los dispositivos del poder político y científico, en muchos casos reactualizando protocolos racistas profundamente arraigados en las significaciones culturales de nuestros países. Se trataba, como tantas veces insiste Enrique Leff, de concebir a la ecología política como fundamentada en una epistemología política, crítica de los conocimientos establecidos por el proyecto de la modernidad, y abriendo la posibilidad para un auténtico diálogo de saberes.

Pero me parece especialmente pertinente rescatar otras dos dimensiones de la perspectiva epistemológica con la que se trabajó en el GT Ecología Política. Por un lado, la propia incorporación de esa denominación, como fundamento de un programa de trabajo, tuvo el mérito de delinear, aunque fuera en sus inicios apenas una convocatoria, una perspectiva unificadora de una problemática teórica. Ya no se trataba de discutir desde la "tierra de nadie" o desde el insoluble laberinto de la soledad de la "sustentabilidad", sino desde un movimiento teórico instituyente de una problemática que partía de considerar la centralidad de las relaciones de poder que estructuran y configuran las relaciones entre sociedades y naturalezas, en toda su complejidad. Problemas que hasta entonces eran tratados dispersamente por la sociología agraria, por la sociología urbana, por el análisis de políticas públicas, por la economía de recursos naturales o por los estudios de movimientos sociales, pasaron a contar con un principio epistemológico organizador, como diferentes manifestaciones de relaciones de poderes bio-políticos sobre la naturaleza, mediadas por los humanos, y sobre

\footnotetext{
${ }^{6}$ Ese grupo de CLACSO tiene continuidad actual, desde 2013, a través del Grupo de Trabajo en Ecología Política del Extractivismo, coordinado por la Dra. Catalina Toro Pérez, de la Universidad Nacional de Colombia.
} 
los humanos, mediadas por la naturaleza, y a través de la producción y reproducción conflictiva de significaciones culturales. Al hacerlo, se podría entonces superar también las limitaciones de escala (por ejemplo, las de la ecología humana tradicional), estableciendo así la visibilidad de lo global en la escala local.

La segunda dimensión que quiero destacar es que este proceso de inserción de la ecología política en las tradiciones del pensamiento latinoamericano no podía dejar de lado la relación fundante con la historia ambiental de nuestra región. La reflexión latinoamericana, desde la independencia, a través de todos sus pensadores, como José Carlos Mariátegui, y en nuestra contemporaneidad,

\section{Referencias}

Alimonda, H. (Comp.). Los Tormentos de la Materia. Buenos Aires: CLACSO, 2005.

Alimonda, H. (Comp.) La Naturaleza Colonizada: Ecología Política y Minería en América Latina. Buenos Aires: CLACSO/ Ciccus, 2011.

Alimonda, H.; Hoetmer, R.; Celestino, D. S. (Eds.). La Amazonía rebelde - Perú 2009. Lima: CLACSO/Cooperación/ CONACAMI/Proyecto Democracia y Transformación Global/ Facultad de Ciencias Sociales, Universidad Nacional Mayor de San Marcos, 2009.

Aricó, J. Marx e a América Latina. Rio de Janeiro: Paz e Terra, 1982.

Bhabha, Homi (Comp.). Nación y narración. Buenos Aires: Siglo XXI, 2010.

Bosi, A. La vanguardia enraizada: el marxismo vivo en Mariátegui. Anuario Mariateguiano, 4(4), 1992.

Delgado Ramos, G. C. ¿Por qué es importante la ecología política? Nueva Sociedad, 244, 2013. como Bolívar Echeverría o Aníbal Quijano, siempre ha destacado la búsqueda de una identidad propia a partir de la indagación en nuestro pasado. No podría proceder de otra manera la formulación de una ecología política latinoamericana, que vendrá a proponer nuevas lecturas de todo nuestro proceso histórico y de la formación de nuestras sociedades a partir de sus claves estratégicas de interpretación. En este sentido, el GT Ecología Política de CLACSO participó con toda propiedad de la creación de la Sociedad Latinoamericana y Caribeña de Historia Ambiental (SOLCHA), en su reunión en La Habana, en 2004.

Escobar, A. Más allá del Tercer Mundo: Globalización y Diferencia. Bogotá: Instituto Colombiano de Antropología e Historia/Universidad del Cauca, 2005.

Jameson, F. Pós-modernismo: a lógica cultural do capitalismo tardio. São Paulo: Ática, 2002.

Kim, S.; Ojo, G. U.; Zaidi, R. Z.; Bryant, R. L. Bringing the other into political ecology: reflecting on preoccupations in a research field. Singapore Journal of Tropical Geography, 33(1), 34-48, 2012. doi: 10.1111/j.1467-9493.2012.00453.x

Martin, F.; Larsimont, R. L'ecologie politique depuis l'Amerique Latine. In: Actes du Premier Colloque sur 'Penser l'ecologie politique: Sciences sociales et interdisciplinarité'. Paris, janvier, 2014.

Martínez Alier, J. Entre la economía ecológica y la ecología política. Sin Permiso, 16 de noviembre, 2014. Disponible en: $<$ http://old.sinpermiso.info/articulos/ficheros/10JMAcol.pdf $>$.

Palacio, G. Is there any "Latin" in the Latin American environmental history? - New challenges for the consolidation of a regional intelectual community. Historia Ambiental Latinoamericana y Caribeña, 1(2), 157, 2012. 